\title{
A GROUP STRUCTURE ON SQUARES
}

\author{
RAVI A. RAO AND SELBY JOSE \\ (Communicated by Paul Goerss)
}

\begin{abstract}
We show that there is an abelian group structure on the orbit set of "squares" of unimodular rows of length $n$ over a commutative ring of stable dimension $d$ when $d=2 n-3, n$ odd and also an abelian group structure on the orbit set of "fourth powers" of unimodular rows of length $n$ over a commutative ring of stable dimension $d$ when $d=2 n-3, n$ even.
\end{abstract}

\section{INTRODUCTION}

L.N. Vaserstein established in [15] a Witt group structure on the orbit space $\mathrm{Um}_{3}(A) / \mathrm{E}_{3}(A)$ of unimodular rows modulo the elementary subgroup, when $A$ has stable dimension two. (Here the stable dimension of $R$ is one less than the stable rank of $R$, where stable rank is as defined in [2], Chapter $5, \S 3$.)

If $R=C(X)$ is the ring of continuous real valued functions on a topological space $X$, then every unimodular row $v \in \mathrm{Um}_{n}(C(X)), n \geq 2$, determines a map $\arg (v): X \longrightarrow \mathbb{R}^{n} \backslash\{0\} \longrightarrow S^{n-1}$. (The first is by evaluation, and the second is the standard homotopy equivalence.) We thus get an element $[\arg (v)]$ of $\left[X, S^{n-1}\right]$. (As $n \geq 2$, we may ignore base points.) Clearly, vectors in the same elementary orbit define homotopic maps. Thus, we have a natural map $\mathrm{Um}_{n}(C(X)) / \mathrm{E}_{n}(C(X)) \longrightarrow$ $\left[X, S^{n-1}\right]=\pi^{n-1}(X)$.

Note that J.F. Adams has shown that $S^{n-1}$ is not an $H$-space (cf. [1]), unless $n=1,2,4$, or 8 . It is classically known that this is equivalent to saying that there is no suitable way to multiply the two projection maps $S^{n-1} \times S^{n-1}$ in $\left[S^{n-1} \times S^{n-1}, S^{n-1}\right]$. However, under suitable restrictions on the "dimension" of $X$ we may expect to define a product on $\pi^{n-1}(X)$.

Henceforth, let $X$ be a finite CW-complex of dimension $d \geq 2$. L.N. Vaserstein has shown in [18] that the ring $C(X)$ has stable dimension $d$. Now let $n \geq 3$, so that $S^{n-1}$ will be at least 1-connected. By the Suspension Theorem, the suspension map $S:\left[X ; S^{n-1}\right] \longrightarrow\left[S X ; S^{n}\right]$ is surjective if $d \leq 2(n-2)+1$, and bijective if $d \leq 2(n-2)$. Moreover, we know that $\left[S X ; S^{n}\right]$ is an abelian group. Hence, the orbit space has a structure of an abelian group if $d \leq 2 n-4$.

Inspired by the group structures on orbits of unimodular rows in the case of rings of continuous functions $C(X)$ on a $\mathrm{CW}$-complex $X, \mathrm{~W}$. van der Kallen was able to obtain similar results algebraically, in the same range. In $[19,20] \mathrm{W}$. van der

Received by the editors October 5, 2006 and, in revised form, January 8, 2007.

2000 Mathematics Subject Classification. Primary 13C10, 15A04, 19G12.

Key words and phrases. Unimodular rows, Suslin matrix, elementary orbit. 
Kallen showed that the orbit space $\operatorname{Um}_{n}(A) / \mathrm{E}_{n}(A)$ has an abelian group structure if $d \leq 2 n-4$, where $d$ is the stable dimension of $A$.

The situation when $d=3, n=3$ is not covered by these estimates, and the results then seem to be different. Let $A=\mathbb{R}[x, y, z, t] /\left(x^{2}+y^{2}+z^{2}+t^{2}-1\right)$ be the 3 -dimensional co-ordinate ring of the real 3 sphere $S^{3}$. In [11] W. van der Kallen and the first named author observed that one could not expect to get a similar Witt group structure on $\mathrm{Um}_{3}(A) / \mathrm{E}_{3}(A)$.

In [12] the first named author showed that the orbit set of "squares" of unimodular 3-rows over a commutative ring $A$, i.e. $\left\{\chi_{2}([v]) \mid v \in \operatorname{Um}_{3}(R)\right\}$, has an abelian group structure, when the stable dimension of $A$ is three. (Here we follow L.N. Vaserstein's notation $\chi_{n}([v])$ in [17].)

Note that by ([20], Theorem 4.1, Lemma $3.5(\mathrm{v}))$ it follows that the orbit set of "squares" is a subgroup of the orbit group $\operatorname{Um}_{n}(R) / \mathrm{E}_{n}(R)$ when $d \leq 2 n-4$, where $d$ is the stable dimension of $R$.

In this short note, we establish that the orbit set of "squares" of unimodular $n$-rows over a commutative ring $A$ has an abelian group structure when $n$ is odd and $d \leq 2 n-3$, where $d$ is the stable dimension of $A$. This reproves and improves the result in [12].

We also show that the orbit set of "fourth powers" of unimodular $n$-vectors over a commutative ring $A$ has an abelian group structure when $n$ is even and $d \leq 2 n-3$, where $d$ is the stable dimension of $A$.

As an application we reprove a special case of a recent result in ([5], Theorem $3.6)$, that if $A$ is an affine algebra of dimension $d$ over an algebraically closed field $k$, with characteristic $k \neq 2$, then the group structure on the orbit space $\mathrm{Um}_{d+1}(A) / \mathrm{E}_{d+1}(A)$ is nice, i.e. $\left[\left(a, z_{1}, \ldots, z_{d}\right)\right] *\left[\left(b, z_{1}, \ldots, z_{d}\right)\right]=\left[\left(a b, z_{1}, \ldots, z_{d}\right)\right]$, for all $\left(a, z_{1}, \ldots, z_{d}\right),\left(b, z_{1}, \ldots, z_{d}\right) \in \operatorname{Um}_{d+1}(A)$. We also show that the group structure on the orbit space $\operatorname{Um}_{d}(A) / \mathrm{E}_{d}(A)$ is nice if $k$ is the algebraic closure of a finite field.

Note that in two cases: $A$ is a non-singular affine algebra of dimension $d$ over an algebraically closed field $k$, or when $A$ is a polynomial extension $R[X]$ of a local ring $R$ of dimension $d$, then every unimodular row of length $d+1$ has a "square" vector in its elementary orbit (cf. [9], [10]). Such results are also expected for unimodular rows of length $d$ over such rings. In particular, one can hope to show that $\mathrm{Um}_{d}(A) / \mathrm{E}_{d}(A)$ has an abelian group structure for such rings, even when $d=2 n-3$, improving on the topological situation.

The Suslin matrices have been used for the first time, in this paper, to obtain a group structure on orbits of some classes of unimodular rows. We feel that this approach can be used in other cases, including recovering the theorems of W. van der Kallen in [19, 20], which generalized results of L.N. Vaserstein in [15], which assert that the orbit space $\operatorname{Um}_{n}(A) / \mathrm{E}_{n}(A)$ has an abelian group structure if $\operatorname{dim}(A)=d \leq 2 n-4$, as well as results of S.M. Bhatwadekar and Raja Sridharan in ([3], Corollary 7.7) and ([4], Corollary 5.9), and later generalized by W. van der Kallen in ([21], Theorem 4.10) that the the image of $\operatorname{Row}_{1}: \operatorname{Um}_{2, n}(A) / \mathrm{E}_{n}(A) \longrightarrow$ $\mathrm{Um}_{n}(A) / \mathrm{E}_{n}(A)$ is a subgroup if $\operatorname{dim}(A) \leq 2 n-5$. More generally, get a group structure on $\mathrm{Um}_{r, n}(A) / \mathrm{E}_{n}(A)$ under suitable restrictions.

That would be a fitting reply to A.A. Suslin's query at the end of ([14], §5). He says that the meaning of the symbols $\operatorname{Um}_{r+1}(A) \longrightarrow \mathrm{G}_{r}(A)$, where $\mathrm{G}_{r}(A)$ is a suitable abelian group, given by associating a pair $(v, w)$ (with $\langle v, w\rangle=1)$ to the 
Suslin matrix $\mathrm{S}_{r}(v, w)$ (recalled in the preliminaries), is unclear and their properties unknown.

\section{Preliminaries}

All rings considered in this article are commutative with 1 .

A row $v=\left(v_{1}, v_{2}, \ldots, v_{n}\right) \in R^{n}$ is said to be unimodular if there exist some elements $w_{1}, w_{2}, \ldots, w_{n}$ in $R$ such that $v_{1} w_{1}+v_{2} w_{2}+\cdots+v_{n} w_{n}=1 . \operatorname{Um}_{n}(R)$ will denote the set of all unimodular rows $v \in R^{n}$.

The group of elementary matrices is a subgroup of $\mathrm{Gl}_{n}(R)$, denoted by $\mathrm{E}_{n}(R)$, and is generated by matrices of the form $E_{i j}(\lambda)=I_{n}+\lambda e_{i j}$, where $\lambda \in R, i \neq j$, $e_{i j} \in \mathrm{M}_{n}(R)$ whose $i j$-th entry is 1 and all other entries are 0 .

$\mathrm{E}_{n}(R)$ acts on $\operatorname{Um}_{n}(R)$ in the natrual way: If $v, w \in \operatorname{Um}_{n}(R)$, then $v \sim_{\mathrm{E}_{n}(R)} w$ means $v=w \varepsilon$ for some $\varepsilon \in \mathrm{E}_{n}(R)$. For simplicity, we denote $\sim_{\mathrm{E}_{n}(R)}$ by $\sim_{E}$.

We shall use the following observations:

Lemma 2.1 ([17], Lemma 1). If $\left(a_{1}, \lambda a_{2}, \lambda a_{3}, a_{4}, \ldots, a_{n}\right) \in \operatorname{Um}_{n}(R), n \geq 3$, for some $\lambda \in R$, then $\left(a_{1}, \lambda a_{2}, \lambda a_{3}, a_{4} \ldots, a_{n}\right) \sim_{E}\left(a_{1}, a_{2}, \ldots, a_{n}\right)$.

Lemma 2.2 ([17], Lemma 4). Let $n \geq 3,\left(a_{1}, a_{2}, \ldots, a_{n}\right) \sim_{E}\left(a_{1}^{\prime}, a_{2}^{\prime}, \ldots, a_{n}^{\prime}\right)$ in $\mathrm{Um}_{n}(R)$ and $m$ be a natural number. Then $\left(a_{1}^{m}, a_{2}, \ldots, a_{n}\right) \sim_{E}\left(a_{1}^{\prime}, a_{2}^{\prime}, \ldots, a_{n}^{\prime}\right)$.

Let $v=\left(a_{1}, a_{2}, \ldots, a_{n}\right)$ be a unimodular row, for some $n \geq 3$. Let $m$ be a natural number. The previous lemma allows one to define $\left.\chi_{m}([v])\right]=\left[\left(a_{1}^{m}, a_{2}, \ldots, a_{n}\right)\right]$. (Note that there is no sanctity to have the power in the first coordinate.)

Lemma 2.3 ([13], Lemma 1). Let $\left(x_{0}, \ldots, x_{r}\right) \in \mathrm{Um}_{r+1}(R), r \geq 2$, and let $t$ be an element of $R$ which is invertible $\bmod \left(R x_{0}+\cdots+R x_{r-2}\right)$. Then $\left(x_{0}, \ldots, x_{r}\right) \sim_{E}$ $\left(x_{0}, \ldots, x_{r-1}, t^{2} x_{r}\right)$.

The Suslin matrix. Given two rows $v, w \in R^{r+1}$, A.A. Suslin in ([14], §5) gave an inductive process to construct the (Suslin) matrix $S_{r}(v, w)$. We recall this process: Let $v=\left(a_{0}, v_{1}\right), w=\left(b_{0}, w_{1}\right)$, where $a_{0}, b_{0} \in R$ and $v_{1}, w_{1} \in \mathrm{M}_{1 r}(R)$. Set $S_{0}(v, w)=a_{0}$, and set

$$
S_{r}(v, w)=\left(\begin{array}{cc}
a_{0} I_{2^{r-1}} & S_{r-1}\left(v_{1}, w_{1}\right) \\
-S_{r-1}\left(w_{1}, v_{1}\right)^{T} & b_{0} I_{2^{r-1}}
\end{array}\right) .
$$

In ([14], Lemma 5.1) it is noted that

$$
S_{r}(v, w) S_{r}(w, v)^{T}=\left(v \cdot w^{T}\right) I_{2^{r}}=S_{r}(w, v)^{T} S_{r}(v, w),
$$

and that $\operatorname{det} S_{r}(v, w)=\left(v \cdot w^{T}\right)^{2^{r-1}}$, for $r \geq 1$.

A.A. Suslin then describes a sequence of forms $J_{r} \in \mathrm{M}_{2^{r}}(R)$ by the recurrence formulae:

$$
J_{r}= \begin{cases}1 & \text { for } r=0, \\ J_{r-1} \perp-J_{r-1}, & \text { for } r \text { even, } \\ J_{r-1} \top-J_{r-1}, & \text { for } r \text { odd. }\end{cases}
$$

(The English translation wrongly says $J_{r}=J_{r-1} \perp J_{r-1}$ when $r$ is even.)

$$
\text { (Here } \alpha \perp \beta=\left(\begin{array}{cc}
\alpha & 0 \\
0 & \beta
\end{array}\right) \text {, while } \alpha \top \beta=\left(\begin{array}{cc}
0 & \alpha \\
\beta & 0
\end{array}\right) \text {.) }
$$

It is easy to see that $\operatorname{det}\left(J_{r}\right)=1$, for all $r$, and that $J_{r}^{T}=J_{r}^{-1}=(-1)^{\frac{r(r+1)}{2}} J_{r}$. Moreover, $J_{r}$ is antisymmetric if $r=4 k+1$ and $r=4 k+2$, whereas $J_{r}$ is symmetric 
for $r=4 k$ and $r=4 k+3$. In ([14], Lemma 5.3), it is noted that the following formulae are valid: The Suslin identities are

$$
\begin{aligned}
\text { for } r=4 k:\left(S_{r}(v, w) J_{r}\right)^{T} & =S_{r}(v, w) J_{r} ; \\
\text { for } r=4 k+1: S_{r}(v, w) J_{r} S_{r}(v, w)^{T} & =\left(v \cdot w^{T}\right) J_{r} ; \\
\text { for } r=4 k+2:\left(S_{r}(v, w) J_{r}\right)^{T} & =-S_{r}(v, w) J_{r} ; \\
\text { for } r=4 k+3: S_{r}(v, w) J_{r} S_{r}(v, w)^{T} & =\left(v \cdot w^{T}\right) J_{r} .
\end{aligned}
$$

Definition. A Suslin matrix $S_{r}(v, w)$ w.r.t. the pair $(v, w)$ is said to be special if $\langle v, w\rangle=v \cdot w^{T}=1$.

Definition. The Special Unimodular Vector group $\operatorname{SUm}_{r}(R)$ is the subgroup of $\mathrm{Sl}_{2^{r}}(R)$ generated by the special Suslin matrices $S_{r}(v, w)$, for all $v, w \in R^{r+1}$, $\langle v, w\rangle=1$.

Definition. The Elementary Unimodular Vector group $\operatorname{EUm}_{r}(R)$ is the subgroup of $\operatorname{SUm}_{r}(R)$ generated by the special Suslin matrices $S_{r}(v, w)$, with $v \in e_{1} \mathrm{E}_{r+1}(R)$, and with $\langle v, w\rangle=1$.

Notation. For a matrix $\alpha \in \mathrm{M}_{k}(R)$, we define $\alpha^{t o p}$ as the matrix whose entries are the same as that of $\alpha$ above the diagonal, and on the diagonal, and is zero below the diagonal. Similarly, we define $\alpha^{\text {bot }}$.

Definition 2.4. Let $v=\left(a_{0}, \ldots, a_{r}\right), w=\left(b_{0}, \ldots, b_{r}\right) \in R^{r+1}$ with $\langle v, w\rangle=1$. The row

$$
v C_{i j}(w, \lambda)=\left(a_{0}, \ldots, a_{i}+\lambda b_{j}, \ldots, a_{j}-\lambda b_{i}, \ldots, a_{r}\right),
$$

for $0 \leq i \neq j \leq r$, is called the Cohn transform of $v$ w.r.t. the vector $w$.

We shall say that a row $v^{*}$ is in the Cohn orbit of $v$ if there is a related row $w^{*}$ to $v^{*}$, and a sequence of pairs, starting with $\left(v_{0}, w_{0}\right)=(v, w)$, and ending with $\left(v_{n}, w_{n}\right)=\left(v^{*}, w^{*}\right)$, such that, for $i \geq 0$, the pairs $\left(v_{i+1}, w_{i+1}\right)$ have either $v_{i+1}$ as a Cohn transform of $v_{i}$ w.r.t. $w_{i}$, and $w_{i+1}=w_{i}$; or $w_{i+1}$ as a Cohn transform of $w_{i}$ w.r.t. $v_{i}$, and $v_{i+1}=v_{i}$ :

$$
(v, w)=\left(v_{0}, w_{0}\right) \rightarrow\left(v_{1}, w_{1}\right) \rightarrow \cdots \rightarrow\left(v_{n}, w_{n}\right)=\left(v^{*}, w^{*}\right) .
$$

Lemma 2.5 ([6], Lemma 2.1). The elementary orbit $v E_{r+1}(R)$ of $v \in \operatorname{Um}_{r+1}(R)$ coincides with the Cohn orbit of $v$, for $r \geq 2$.

We now state the Key Lemma of [6].

Lemma 2.6 ([6], Lemma 3.2). Let $v, w \in R^{r+1}$, with $v \cdot w^{T}=1$. Then, for $r \geq 2$, $2 \leq i \leq r+1, \lambda \in R$,

$$
\begin{aligned}
& S_{r}\left(e_{1} E_{1 i}(\lambda), e_{1}\right)^{b o t} S_{r}(v, w) S_{r}\left(e_{1} E_{1 i}(\lambda), e_{1}\right)^{t o p}=S_{r}\left(v E_{1 i}(\lambda), w E_{i 1}(-\lambda)\right), \\
& S_{r}\left(e_{1}, e_{1} E_{1 i}(\lambda)\right)^{t o p} S_{r}(v, w) S_{r}\left(e_{1}, e_{1} E_{1 i}(\lambda)\right)^{b o t}=S_{r}\left(v E_{i 1}(-\lambda), w E_{1 i}(\lambda)\right), \\
& S_{r}\left(e_{1} E_{1 i}(\lambda), e_{1}\right)^{t o p} S_{r}(v, w) S_{r}\left(e_{1} E_{1 i}(\lambda), e_{1}\right)^{b o t}=S_{r}\left(v C_{0 i-1}(-\lambda), w\right), \\
& S_{r}\left(e_{1}, e_{1} E_{1 i}(\lambda)\right)^{b o t} S_{r}(v, w) S_{r}\left(e_{1}, e_{1} E_{1 i}(\lambda)\right)^{t o p}=S_{r}\left(v, w C_{0 i-1}(-\lambda)\right) .
\end{aligned}
$$

(The case when $r=1$ is similar.) 
Lemma 2.7 ([8], Lemma 4.6). One has the following relations in $\operatorname{EUm}_{r}(R)$ : For $2 \leq i \neq j \leq r+1, \lambda \in R$,

$$
\begin{aligned}
S_{r}\left(e_{1} E_{1 i}(\lambda), e_{1}\right)^{t o p} & \\
= & S_{r}\left(e_{1} E_{1 j}(\lambda), e_{1}\right) S_{r}\left(e_{1}-\lambda e_{j}+e_{i}, e_{1}\right) S_{r}\left(e_{1} E_{1 i}(-1), e_{1}\right) \\
& S_{r}\left((1-\lambda) e_{1}+\lambda e_{j}+e_{i}, e_{1}+e_{j}\right) S_{r}\left(e_{1}-e_{i}, e_{1}-e_{j}\right) \\
& S_{r}\left((1+\lambda) e_{1}-\lambda e_{j}, e_{1}+e_{j}\right) S_{r}\left(e_{1}, e_{1} E_{1 j}(-1)\right),
\end{aligned}
$$

$S_{r}\left(e_{1}, e_{1} E_{1 i}(\lambda)\right)^{t o p}$

$$
\begin{aligned}
= & S_{r}\left(e_{1}+\lambda e_{j}, e_{1}+e_{i}\right) S_{r}\left(e_{1} E_{1 j}(-\lambda), e_{1}\right) S_{r}\left(e_{1}, e_{1} E_{1 i}(-1)\right) \\
& S_{r}\left(e_{1}, e_{1}+e_{i}+e_{j}\right) S_{r}\left(e_{1}+\lambda e_{j},(1+\lambda) e_{1}-e_{i}-e_{j}\right) \\
& S_{r}\left((1+\lambda) e_{1}-\lambda e_{j}, e_{1}+e_{j}\right) S_{r}\left(e_{1}, e_{1} E_{1 j}(-1)\right) .
\end{aligned}
$$

(Note that by reversing the order of the elements in the product in the above relation we obtain the formulae for $S_{r}\left(e_{1} E_{1 i}(\lambda), e_{1}\right)^{\text {bot }}$ and $S_{r}\left(e_{1}, e_{1} E_{1 i}(\lambda)\right)^{b o t}$.)

Theorem 2.8 ([6], Proposition 5.6, Theorem 5.8, [7], Proposition 2.6). The elements

$$
\begin{aligned}
& S_{r}\left(e_{1} E_{1 i}(\lambda), e_{1}\right)^{t o p}, \\
& S_{r}\left(e_{1} E_{1 i}(\lambda), e_{1}\right)^{b o t}, \\
& S_{r}\left(e_{1}, e_{1} E_{1 i}(\lambda)\right)^{t o p}, \\
& S_{r}\left(e_{1}, e_{1} E_{1 i}(\lambda)\right)^{b o t},
\end{aligned}
$$

for $2 \leq i \leq r+1, \lambda \in R$, belong to $\operatorname{EUm}_{r}(R)$. Moreover they generate the group $\operatorname{EUm}_{r}(R)$

An involution $*$ on $\mathbf{S U m}_{r}(R), r$ even. Let $\alpha=\prod_{i=1}^{n} S_{i}$ be a product of Suslin matrices $S_{i}=S_{r}\left(v_{i}, w_{i}\right)$, and let $\alpha^{\vee}$ denote $\prod_{i=n}^{1} S_{i}$. (A priori $\alpha^{\vee}$ will depend on the splitting of $\alpha$.) If $r$ is even, then $\alpha \mapsto \alpha^{\vee}$ is a well defined anti-involution on $\mathrm{SUm}_{r}(R)$ : By Suslin identities,

$$
S_{r}(v, w)=J_{r} S_{r}(v, w)^{T} J_{r}^{-1} .
$$

Hence, $\alpha^{\vee}=J_{r} \alpha^{T} J_{r}^{-1}$ only depends on $\alpha$. Thus if $\alpha=S_{1} \ldots S_{n}=S_{1}^{\prime} \ldots S_{m}^{\prime} \in$ $\operatorname{SUm}_{r}(R)$, for some special Suslin matrices $S_{1}, \ldots, S_{n}, S_{1}^{\prime}, \ldots, S_{m}^{\prime}$, if $r$ is even, then $S_{m}^{\prime} \ldots S_{1}^{\prime}=S_{n} \ldots S_{1}$. We shall denote $\alpha^{\vee}$ by $\alpha^{*}$ to emphasize that it is independent of the splitting chosen.

The situation is different when $r$ is odd. In this case one has

Lemma 2.9 ([8], Corollary 3.2). Let $S_{i}=S_{r}\left(v_{i}, w_{i}\right)$, for $1 \leq i \leq n$, be special Suslin matrices. Let $\alpha=S_{1} \ldots S_{n}, \alpha^{\vee}=S_{n} \ldots S_{1}$. If $\alpha=I_{2^{r}}, r>1$ odd, then $\alpha^{\vee}=u I_{2^{r}}$, with $u^{2}=1$. (If $r$ is even, then $\alpha^{\vee}=I_{2^{r}}$.)

In $([8], \S 5)$ an example is given to show that $\alpha^{\vee}$ does depend (up to a unit factor $u$, with $u^{2}=1$ ) on the splitting chosen of $\alpha$, when $r$ is odd.

Remark 2.10. If one takes the splitting prescribed in Lemma 2.7, one gets the following:

(i) If $\alpha=S_{r}\left(e_{1} E_{1 i}(\lambda), e_{1}\right)^{t o p}$, then $\alpha^{*}=S_{r}\left(e_{1} E_{1 i}(\lambda), e_{1}\right)^{b o t}=\alpha^{\vee}$.

(ii) If $\beta=S_{r}\left(e_{1} E_{1 i}(\lambda), e_{1}\right)^{b o t}$, then $\beta^{*}=S_{r}\left(e_{1} E_{1 i}(\lambda), e_{1}\right)^{t o p}=\beta^{\vee}$.

(iii) If $\gamma=S_{r}\left(e_{1}, e_{1} E_{1 i}(\lambda)\right)^{t o p}$, then $\gamma^{*}=S_{r}\left(e_{1}, e_{1} E_{1 i}(\lambda)\right)^{b o t}=\gamma^{\vee}$.

(iv) If $\delta=S_{r}\left(e_{1}, e_{1} E_{1 i}(\lambda)\right)^{b o t}$, then $\delta^{*}=S_{r}\left(e_{1}, e_{1} E_{1 i}(\lambda)\right)^{t o p}=\delta^{\vee}$. 
Corollary 2.11. Let $\alpha \in \operatorname{EUm}_{r}(R), r>1$. Then

(a) if $r$ is even, $\alpha S_{r}(v, w) \alpha^{*}=S_{r}\left(v \varepsilon, w \varepsilon^{T^{-1}}\right)$, for some $\varepsilon \in \mathrm{E}_{r+1}(R)$,

(b) if $r$ is odd, $\alpha S_{r}(v, w) \alpha^{\vee}=S_{r}\left(v \varepsilon, w \varepsilon^{T^{-1}}\right)$, for some $\varepsilon \in \mathrm{E}_{r+1}(R)$.

Proof. By Theorem 2.8, we can write $\alpha$ as a product of elements of the type $S_{r}\left(e_{1} E_{1 i}(\lambda), e_{1}\right)^{t o p}, S_{r}\left(e_{1} E_{1 i}(\lambda), e_{1}\right)^{b o t}, S_{r}\left(e_{1}, e_{1} E_{1 i}(\lambda)\right)^{t o p}$ and $S_{r}\left(e_{1}, e_{1} E_{1 i}(\lambda)\right)^{b o t}$. Now apply Key Lemma 2.6, and note that by Lemma 2.5, the Cohn orbit is the same as the elementary orbit.

(b) is proved similarly. One only has to realize by Lemma 2.1 that $u v \sim_{E} v$, if $u$ is a unit and $v \in \operatorname{Um}_{r+1}(R)$ (being of even length).

\section{The group structure}

We prove that the set of "squares" $\chi_{2}([v])$ in the orbit set $\operatorname{Um}_{r+1}(R) / \mathrm{E}_{r+1}(R)$ (denoted by $\operatorname{SqUm}_{r+1}(R) / \mathrm{E}_{r+1}(R)$ )

$$
\begin{aligned}
& =\left\{\chi_{2}([v]): v \in \mathrm{Um}_{r+1}(R)\right\} \\
& =\left\{\left[\left(a_{0}^{2}, a_{1}, \ldots, a_{r}\right)\right]:\left(a_{0}, a_{1}, \ldots, a_{r}\right) \in \mathrm{Um}_{r+1}(R)\right\}
\end{aligned}
$$

has an abelian group structure if $r$ is even and $2 r \geq d+1$. We also prove that the set of "fourth powers" $\chi_{4}([v])$ in the orbit set $\operatorname{Um}_{r+1}(R) / \mathrm{E}_{r+1}(R)$ (denoted by $\left.\operatorname{SqSqUm}_{r+1}(R) / \mathrm{E}_{r+1}(R)\right)$

$$
\begin{aligned}
& =\left\{\chi_{4}([v]): v \in \mathrm{Um}_{r+1}(R)\right\} \\
& =\left\{\left[\left(a_{0}^{4}, a_{1}, \ldots, a_{r}\right)\right]:\left(a_{0}, a_{1}, \ldots, a_{r}\right) \in \mathrm{Um}_{r+1}(R)\right\}
\end{aligned}
$$

has an abelian group structure if $r$ is odd and $2 r \geq d+1$. The main reason for the restriction on the size is due to the following lemma.

Lemma 3.1 ((Mennicke-Newman) [21], Lemma 3.2). Let $R$ be a commutative ring of stable dimension less than or equal to $2 n-3$ for some $n \geq 3$. Suppose that finitely many orbits under $\mathrm{E}_{n}(R)$ are given in $\operatorname{Um}_{n}(R)$. Then one can choose orbit representatives in such a way that for any two orbits, the chosen representatives differ at most in their first coordinate.

We now prove the lemma which is needed to establish the group structure.

Lemma 3.2. Let $S_{r}(v, w), S_{r}(p, q), S_{r}\left(v^{\prime}, w^{\prime}\right), S_{r}\left(p^{\prime}, q^{\prime}\right)$ be special Suslin matrices, where $v=\left(a_{0}, a_{1}, v_{1}\right), w=\left(b_{0}, b_{1}, w_{1}\right), p=\left(c_{0}, a_{1}, v_{1}\right), q=\left(d_{0}, b_{1}, w_{1}\right)$ $v^{\prime}=\left(a_{0}, a_{1}^{2}, v_{1}\right), w^{\prime}=\left(b_{0}, b_{1}^{\prime}, w_{1}\right), p^{\prime}=\left(c_{0}, a_{1}^{2}, v_{1}\right), q^{\prime}=\left(d_{0}, b_{1}^{\prime}, w_{1}\right) \in \operatorname{Um}_{r+1}(R)$, $a_{0}, a_{1}, b_{0}, b_{1}, b_{1}^{\prime}, c_{0}, d_{0} \in R$, and $v_{1}, w_{1} \in \mathrm{M}_{1 r-1}(R)$. Then there exist $\varepsilon_{1}, \varepsilon_{2} \in$ $\operatorname{EUm}_{r}(R)$, such that

(i) $\varepsilon_{1} S_{r}(p, q) S_{r}(v, w) S_{r}(p, q) \varepsilon_{1}^{*}=S_{r}\left(\left(a_{0} c_{0}^{2}, a_{1}, v_{1}\right)\right.$, w1 $w_{1}^{\prime}$, where $w_{1}^{\prime}$ is such that $\left\langle\left(a_{0} c_{0}^{2}, a_{1}, v_{1}\right), w_{1}^{\prime}\right\rangle=1$, if $r$ is even, and

(ii) $\varepsilon_{2} S_{r}\left(p^{\prime}, q^{\prime}\right) S_{r}\left(v^{\prime}, w^{\prime}\right) S_{r}\left(p^{\prime}, q^{\prime}\right) \varepsilon_{2}^{\vee}=S_{r}\left(\left(a_{0} c_{0}^{2}, a_{1}^{2}, v_{1}\right), w^{\prime \prime}\right)$, where $w^{\prime \prime}$ is such that $\left\langle\left(a_{0} c_{0}^{2}, a_{1}^{2}, v_{1}\right), w^{\prime \prime}\right\rangle=1$, if $r$ is odd.

Proof. Let $S_{r}(v, w)=\left(\begin{array}{cc}a_{0} & S \\ T & b_{0}\end{array}\right)$ and $S_{r}(p, q)=\left(\begin{array}{cc}c_{0} & S \\ T & d_{0}\end{array}\right)$, where

$$
S=S_{r-1}\left(\left(a_{1}, v_{1}\right),\left(b_{1}, w_{1}\right)\right) \quad \text { and } \quad T=-S_{r-1}\left(\left(b_{1}, w_{1}\right),\left(a_{1}, v_{1}\right)\right)^{T} \text {. }
$$


By direct computation, one gets

$$
\begin{aligned}
S_{r}(p, q) & S_{r}(v, w) S_{r}(p, q) \\
= & \left(\begin{array}{cc}
\left(a_{0} c_{0}^{2}+\beta S T\right) I_{2^{r-1}} & \lambda S \\
\lambda T & \left(b_{0} d_{0}^{2}+\gamma S T\right) I_{2^{r-1}}
\end{array}\right) \\
\quad= & S_{r}\left(\left(a_{0} c_{0}^{2}+\beta S T, \lambda a_{1}, \lambda v_{1}\right),\left(b_{0} d_{0}^{2}+\gamma S T, \lambda b_{1}, \lambda w_{1}\right)\right),
\end{aligned}
$$

for some $\beta, \gamma, \lambda \in R$.

Note that $1=a_{0} b_{0}+a_{1} b_{1}-S T=a_{0} b_{0}+a_{1} b_{1}+\left\langle v_{1}, w_{1}\right\rangle$. If $r$ is even, by Lemma 2.1,

$$
\left(a_{0} c_{0}^{2}+\beta S T, \lambda a_{1}, \lambda v_{1}\right) \sim_{E}\left(a_{0} c_{0}^{2}+\beta S T, a_{1}, v_{1}\right) \sim_{E}\left(a_{0} c_{0}^{2}, a_{1}, v_{1}\right) .
$$

Thus by Key Lemma 2.6, there exists an $\varepsilon_{1} \in \operatorname{EUm}_{r}(R)$ such that

$$
S_{r}(p, q) S_{r}(v, w) S_{r}(p, q)=\varepsilon_{1} S_{r}\left(\left(a_{0} c_{0}^{2}, a_{1}, v_{1}\right), w_{1}^{\prime}\right) \varepsilon_{1}^{*}
$$

where $w_{1}^{\prime}$ is such that $\left\langle\left(a_{0} c_{0}^{2}, a_{1}, v_{1}\right), w_{1}^{\prime}\right\rangle=1$.

Note that $\left(a_{0}^{2}, a_{1}, v_{1}\right) \sim_{E}\left(a_{0}, a_{1}^{2}, v_{1}\right)$ and $\left(c_{0}^{2}, a_{1}, v_{1}\right) \sim_{E}\left(c_{0}, a_{1}^{2}, v_{1}\right)$. Let $S_{r}\left(v^{\prime}, w^{\prime}\right)$ $=\left(\begin{array}{ll}a_{0} & S_{1} \\ T_{1} & b_{0}\end{array}\right)$ and $S_{r}\left(p^{\prime}, q^{\prime}\right)=\left(\begin{array}{cc}c_{0} & S_{1} \\ T_{1} & d_{0}\end{array}\right)$, where $S=S_{r-1}\left(\left(a_{1}^{2}, v_{1}\right),\left(b_{1}^{\prime}, w_{1}\right)\right)$ and $T=-S_{r-1}\left(\left(b_{1}^{\prime}, w_{1}\right),\left(a_{1}^{2}, v_{1}\right)\right)^{T}$. By direct computation, one gets

$$
\begin{aligned}
S_{r}\left(p^{\prime}, q^{\prime}\right) S_{r}\left(v^{\prime}, w^{\prime}\right) S_{r}\left(p^{\prime}, q^{\prime}\right) & \\
\quad= & \left(\begin{array}{cc}
\left(a_{0} c_{0}^{2}+\beta_{1} S_{1} T_{1}\right) I_{2^{r-1}} & \lambda_{1} S_{1} \\
\lambda_{1} T_{1} & \left(b_{0} d_{0}^{2}+\gamma_{1} S_{1} T_{1}\right) I_{2^{r-1}}
\end{array}\right) \\
\quad= & S_{r}\left(\left(a_{0} c_{0}^{2}+\beta_{1} S_{1} T_{1}, \lambda_{1} a_{1}^{2}, \lambda_{1} v_{1}\right),\left(b_{0} d_{0}^{2}+\gamma_{1} S_{1} T_{1}, \lambda_{1} b_{1}^{\prime}, \lambda_{1} w_{1}\right)\right),
\end{aligned}
$$

for some $\beta_{1}, \gamma_{1}, \lambda_{1} \in R$.

If $r$ is odd, via. Lemma 2.1, Lemma 2.2 and Lemma 2.3,

$$
\begin{aligned}
\left(a_{0} c_{0}^{2}+\beta_{1} S_{1} T_{1}, \lambda_{1} a_{1}^{2}, \lambda_{1} v_{1}\right) & =\left(a_{0} c_{0}^{2}+\beta_{1} S_{1} T_{1}, \lambda_{1} a_{1}^{2}, \lambda_{1} a_{2}, \lambda_{1} a_{3}, \ldots, \lambda_{1} a_{r}\right) \\
& \sim_{E}\left(a_{0} c_{0}^{2}+\beta_{1} S_{1} T_{1}, a_{1}^{2}, \lambda_{1} a_{2}, a_{3}, \ldots, a_{r}\right) \\
& \sim_{E}\left(a_{0} c_{0}^{2}+\beta_{1} S_{1} T_{1}, a_{1},\left(\lambda_{1} a_{2}\right)^{2}, a_{3}, \ldots, a_{r}\right) \\
& \sim_{E}\left(a_{0} c_{0}^{2}+\beta_{1} S_{1} T_{1}, a_{1}, \lambda_{1}^{2} a_{2}^{2}, a_{3}, \ldots, a_{r}\right) \\
& \sim_{E}\left(a_{0} c_{0}^{2}+\beta_{1} S_{1} T_{1}, a_{1}, a_{2}^{2}, a_{3}, \ldots, a_{r}\right) \\
& \sim_{E}\left(a_{0} c_{0}^{2}+\beta_{1} S_{1} T_{1}, a_{1}^{2}, a_{2}, a_{3}, \ldots, a_{r}\right) \\
& \sim_{E}\left(a_{0} c_{0}^{2}, a_{1}^{2}, a_{2}, a_{3}, \ldots, a_{r}\right) .
\end{aligned}
$$

Thus by Key Lemma 2.6, there exists an $\varepsilon_{2} \in \operatorname{EUm}_{r}(R)$ such that

$$
S_{r}\left(p^{\prime}, q^{\prime}\right) S_{r}\left(v^{\prime}, w^{\prime}\right) S_{r}\left(p^{\prime}, q^{\prime}\right)=\varepsilon_{2} S_{r}\left(\left(a_{0} c_{0}^{2}, a_{1}^{2}, v_{1}\right), w^{\prime \prime}\right) \varepsilon_{2}^{\vee}
$$

where $w^{\prime \prime}$ is such that $\left\langle\left(a_{0} c_{0}^{2}, a_{1}^{2}, v_{1}\right), w^{\prime \prime}\right\rangle=1$.

Lemma 3.3. Let $a_{0}, a_{1}, a_{0}^{\prime}, a_{1}^{\prime}, c_{0}, c_{0}^{\prime} \in R, v_{1}, v_{1}^{\prime} \in M_{1 r-1}(R)$.

(i) If $r$ is even and $\left(a_{0}, a_{1}, v_{1}\right) \sim_{E}\left(a_{0}^{\prime}, a_{1}^{\prime}, v_{1}^{\prime}\right),\left(c_{0}, a_{1}, v_{1}\right) \sim_{E}\left(c_{0}^{\prime}, a_{1}^{\prime}, v_{1}^{\prime}\right)$ in $\operatorname{Um}_{r+1}(R)$, then $\left(a_{0} c_{0}^{2}, a_{1}, v_{1}\right) \sim_{E}\left(a_{0}^{\prime} c_{0}^{\prime 2}, a_{1}^{\prime}, v_{1}^{\prime}\right)$.

(ii) If $r$ is odd and $\left(a_{0}, a_{1}^{2}, v_{1}\right) \sim_{E}\left(a_{0}^{\prime}, a_{1}^{\prime 2}, v_{1}^{\prime}\right),\left(c_{0}, a_{1}^{2}, v_{1}\right) \sim_{E}\left(c_{0}^{\prime}, a_{1}^{\prime 2}, v_{1}^{\prime}\right)$ in $\mathrm{Um}_{r+1}(R)$, then $\left(a_{0} c_{0}^{2}, a_{1}^{2}, v_{1}\right) \sim_{E}\left(a_{0}^{\prime} c_{0}^{\prime 2}, a_{1}^{\prime 2}, v_{1}^{\prime}\right)$. 
Proof. Let $r$ be even. Via Key Lemma 2.6, it is enough to prove that if there are $\varepsilon_{1}, \varepsilon_{2} \in \mathrm{EUm}_{r}(R)$ such that

$$
\begin{aligned}
& \varepsilon_{1} S_{r}\left(\left(a_{0}, a_{1}, v_{1}\right), w_{1}\right) \varepsilon_{1}^{*}=S_{r}\left(\left(a_{0}^{\prime}, a_{1}^{\prime}, v_{1}^{\prime}\right), w_{1}^{\prime}\right), \\
& \varepsilon_{2} S_{r}\left(\left(c_{0}, a_{1}, v_{1}\right), w_{1}\right) \varepsilon_{2}^{*}=S_{r}\left(\left(c_{0}^{\prime}, a_{1}^{\prime}, v_{1}^{\prime}\right), w_{1}^{\prime}\right),
\end{aligned}
$$

then there exists an $\varepsilon_{3} \in \operatorname{EUm}_{r}(R)$ such that

$$
\varepsilon_{3} S_{r}\left(\left(a_{0} c_{0}^{2}, a_{1}, v_{1}\right), w_{2}\right) \varepsilon_{3}^{*}=S_{r}\left(\left(a^{\prime} c^{\prime 2}, a_{1}^{\prime}, v_{1}^{\prime}\right), w_{2}^{\prime}\right) .
$$

By the normality of $\operatorname{EUm}_{r}(R)$ in $\operatorname{SUm}_{r}(R)$ (see [7], Corollary 3.6)

$$
\begin{aligned}
& S_{r}\left(\left(c_{0}, a_{1}, v_{1}\right), w_{1}\right) S_{r}\left(\left(a_{0}, a_{1}, v_{1}\right), w_{1}\right) S_{r}\left(\left(c_{0}, a_{1}, v_{1}\right), w_{1}\right) \\
& \quad=\varepsilon^{\prime} S_{r}\left(\left(c_{0}^{\prime}, a_{1}^{\prime}, v_{1}^{\prime}\right), w_{1}^{\prime}\right) S_{r}\left(\left(a_{0}^{\prime}, a_{1}^{\prime}, v_{1}^{\prime}\right), w_{1}^{\prime}\right) S_{r}\left(\left(c_{0}^{\prime}, a_{1}^{\prime}, v_{1}^{\prime}\right), w_{1}^{\prime}\right) \varepsilon^{\prime *},
\end{aligned}
$$

for some $\varepsilon^{\prime} \in \operatorname{EUm}_{r}(R)$.

By Lemma 3.2, there exists $\varepsilon_{4}, \varepsilon_{5} \in \operatorname{EUm}_{r}(R)$ such that

$$
\begin{aligned}
& S_{r}\left(\left(c_{0}, a_{1}, v_{1}\right), w_{1}\right) S_{r}\left(\left(a_{0}, a_{1}, v_{1}\right), w_{1}\right) S_{r}\left(\left(c_{0}, a_{1}, v_{1}\right), w_{1}\right) \\
& =\varepsilon_{4} S_{r}\left(\left(a_{0} c_{0}^{2}, a_{1}, v_{1}\right), w_{1}^{\prime \prime}\right) \varepsilon_{4}^{*}, \\
& S_{r}\left(\left(c_{0}^{\prime}, a_{1}^{\prime}, v_{1}^{\prime}\right), w_{1}^{\prime}\right) S_{r}\left(\left(a_{0}^{\prime}, a_{1}^{\prime}, v_{1}^{\prime}\right), w_{1}^{\prime}\right) S_{r}\left(\left(c_{0}^{\prime}, a_{1}^{\prime}, v_{1}^{\prime}\right), w_{1}^{\prime}\right) \\
& =\varepsilon_{5} S_{r}\left(\left(a_{0}^{\prime} c_{0}^{\prime 2}, a_{1}^{\prime}, v_{1}^{\prime}\right), w_{2}^{\prime \prime}\right) \varepsilon_{5}^{*} .
\end{aligned}
$$

Thus $\varepsilon S_{r}\left(\left(a_{0} c_{0}^{2}, a_{1}, v_{1}\right), w_{1}^{\prime \prime}\right) \varepsilon^{*}=S_{r}\left(\left(a_{0}^{\prime} c_{0}^{\prime 2}, a_{1}^{\prime}, v_{1}^{\prime}\right), w_{2}^{\prime \prime}\right)$ for some $\varepsilon \in \operatorname{EUm}_{r}(R)$. By Key Lemma 2.6,

$$
\varepsilon S_{r}\left(\left(a_{0} c_{0}^{2}, a_{1}, v_{1}\right), w_{1}\right) \varepsilon^{*}=S_{r}\left(\left(a_{0} c_{0}^{2}, a_{1}, v_{1}\right) \varepsilon_{6}, w_{1} \varepsilon_{6}^{T^{-1}}\right),
$$

for some $\varepsilon_{6} \in \mathrm{E}_{r+1}(R)$. Hence $\left(a_{0} c_{0}^{2}, a_{1}, v_{1}\right) \sim_{E}\left(a_{0}^{\prime} c_{0}^{\prime 2}, a_{1}^{\prime}, v_{1}^{\prime}\right)$.

When $r$ is odd, by the same argument as above, one gets

$$
\varepsilon_{7} S_{r}\left(\left(a_{0} c_{0}^{2}, a_{1}^{2}, v_{1}\right), w_{3}\right) \varepsilon_{7}^{\vee}=S_{r}\left(\left(a_{0} c_{0}^{2}, a_{1}^{2}, v_{1}\right) \varepsilon_{8}, w_{1} \varepsilon_{8}^{T^{-1}}\right),
$$

for some $\varepsilon_{7} \in \operatorname{EUm}_{r}(R)$ and $\varepsilon_{8} \in \mathrm{E}_{r+1}(R)$. Hence $\left(a_{0} c_{0}^{2}, a_{1}^{2}, v_{1}\right) \sim_{E}\left(a_{0}^{\prime} c_{0}^{\prime 2}, a_{1}^{\prime 2}, v_{1}^{\prime}\right)$.

Note that $\varepsilon_{7}^{\vee}$ depends on the splitting one chooses of $\varepsilon_{7}$ and by Lemma 2.9, if one changes the splitting of $\varepsilon_{7}$ and reverses it. Then one gets,

$$
\varepsilon_{7} S_{r}\left(\left(a_{0} c_{0}^{2}, a_{1}^{2}, v_{1}\right), w_{3}\right) \varepsilon_{7}^{\vee}=u S_{r}\left(\left(a_{0} c_{0}^{2}, a_{1}^{2}, v_{1}\right) \varepsilon_{8}, w_{1} \varepsilon_{8}^{T^{-1}}\right),
$$

for some unit $u \in R$ with $u^{2}=1$. By Lemma 2.1 and Key Lemma 2.6, as $r$ is odd, one gets

$$
\varepsilon_{7}^{\prime \prime} S_{r}\left(\left(a_{0} c_{0}^{2}, a_{1}^{2}, v_{1}\right) \varepsilon_{8}, w_{1} \varepsilon_{8}^{T^{-1}}\right) \varepsilon_{7}^{\prime \prime \vee}=u S_{r}\left(\left(a_{0} c_{0}^{2}, a_{1}^{2}, v_{1}\right) \varepsilon_{8}, w_{1} \varepsilon_{8}^{T^{-1}}\right),
$$

for some $\varepsilon_{7}^{\prime \prime} \in \operatorname{EUm}_{r}(R)$.

By Lemma 3.1 it suffices to define the product $\chi_{2}([v]) * \chi_{2}\left(\left[v^{\prime}\right]\right)$ and $\chi_{4}([v]) * \chi_{4}\left(\left[v^{\prime}\right]\right)$, where $v$ and $v^{\prime}$ differ at most in their first coordinates. We propose that the binary operation $*$ on $\operatorname{SqUm}_{r+1}(R) / \mathrm{E}_{r+1}(R)$ is defined as

$$
\left[\left(a_{0}^{2}, a_{1}, v_{1}\right)\right] *\left[\left(c_{0}^{2}, a_{1}, v_{1}\right)\right]=\left[\left(a_{0}^{2} c_{0}^{2}, a_{1}, v_{1}\right)\right]
$$

and the binary operation $*^{\prime}$ on $\operatorname{SqSqUm}_{r+1}(R) / \mathrm{E}_{r+1}(R)$ is defined as

$$
\left[\left(a_{0}^{2}, a_{1}^{2}, v_{1}\right)\right] *^{\prime}\left[\left(c_{0}^{2}, a_{1}^{2}, v_{1}\right)\right]=\left[\left(a_{0}^{2} c_{0}^{2}, a_{1}^{2}, v_{1}\right)\right]
$$

where $v_{1} \in \mathrm{M}_{1 r-1}(R), a_{0}, a_{1}, c_{0} \in R$. 
We shall establish that $*$ and $*^{\prime}$ are well defined operations. After that it is easy to see (via Lemma 3.1) that $*$ and $*^{\prime}$ are associative. If $a_{0} b_{0}+a_{1} b_{1}+\cdots+$ $a_{r} b_{r}=1$, then it is easy to check that $\left[\left(a_{0}^{2}, a_{1}, \ldots, a_{r}\right)\right]^{-1}=\left[\left(b_{0}^{2}, a_{1}, \ldots, a_{r}\right)\right]$ and $\left[\left(a_{0}^{2}, a_{1}^{2}, \ldots, a_{r}\right)\right]^{-1}=\left[\left(b_{0}^{2}, a_{1}^{2}, \ldots, a_{r}\right)\right]$. Hence one has an abelian group structure on $\operatorname{SqUm}_{r+1}(R) / \mathrm{E}_{r+1}(R)$ and $\operatorname{SqSqUm}_{r+1}(R) / \mathrm{E}_{r+1}(R)$.

Lemma 3.4. Let $a_{0}, a_{1}, x_{0}, b_{1}, c_{0}, y_{0} \in R, v_{1}, w_{1} \in M_{1 r-1}(R)$.

(i) If $r$ is even, $\left(a_{0}^{2}, a_{1}, v_{1}\right) \sim_{E}\left(x_{0}^{2}, b_{1}, w_{1}\right),\left(c_{0}^{2}, a_{1}, v_{1}\right) \sim_{E}\left(y_{0}^{2}, b_{1}, w_{1}\right)$ in $\operatorname{Um}_{r+1}(R)$, then $\left(a_{0}^{2} c_{0}^{2}, a_{1}, v_{1}\right) \sim_{E}\left(x_{0}^{2} y_{0}^{2}, b_{1}, w_{1}\right)$.

(ii) If $r$ is odd, $\left(a_{0}^{2}, a_{1}^{2}, v_{1}\right) \sim_{E}\left(x_{0}^{2}, b_{1}^{2}, w_{1}\right),\left(c_{0}^{2}, a_{1}^{2}, v_{1}\right) \sim_{E}\left(y_{0}^{2}, b_{1}^{2}, w_{1}\right)$ in $\operatorname{Um}_{r+1}(R)$, then $\left(a_{0}^{2} c_{0}^{2}, a_{1}^{2}, v_{1}\right) \sim_{E}\left(x_{0}^{2} y_{0}^{2}, b_{1}^{2}, w_{1}\right)$.

Consequently, $*$ and $*^{\prime}$ are well defined operations.

Proof. Suppose $r$ is even. By Mennicke-Newman Lemma 3.1, one can arrange

$$
\begin{array}{ll}
\left(a_{0}, a_{1}, v_{1}\right) \sim_{E}\left(p_{0}, p_{1}, v\right), & \left(x_{0}, b_{1}, w_{1}\right) \sim_{E}\left(q_{0}, p_{1}, v\right), \\
\left(c_{0}, a_{1}, v_{1}\right) \sim_{E}\left(s_{0}, p_{1}, v\right) \text { and } & \left(y_{0}, b_{1}, w_{1}\right) \sim_{E}\left(t_{0}, p_{1}, v\right) .
\end{array}
$$

By Lemma 2.2,

$$
\begin{array}{ll}
\left(a_{0}^{2}, a_{1}, v_{1}\right) \sim_{E}\left(p_{0}^{2}, p_{1}, v\right), & \left(x_{0}^{2}, b_{1}, w_{1}\right) \sim_{E}\left(q_{0}^{2}, p_{1}, v\right), \\
\left(c_{0}^{2}, a_{1}, v_{1}\right) \sim_{E}\left(s_{0}^{2}, p_{1}, v\right) \text { and } & \left(y_{0}^{2}, b_{1}, w_{1}\right) \sim_{E}\left(t_{0}^{2}, p_{1}, v\right) .
\end{array}
$$

Since $\left(a_{0}^{2}, a_{1}, v_{1}\right) \sim_{E}\left(p_{0}^{2}, p_{1}, v\right)$ and $\left(c_{0}, a_{1}, v_{1}\right) \sim_{E}\left(s_{0}, p_{1}, v\right)$, one has by Lemma $3.3(\mathrm{i})$,

$$
\left(a_{0}^{2} c_{0}^{2}, a_{1}, v_{1}\right) \sim_{E} \quad\left(p_{0}^{2} s_{0}^{2}, p_{1}, v\right) .
$$

Also as $\left(x_{0}^{2}, b_{1}, w_{1}\right) \sim_{E}\left(q_{0}^{2}, p_{1}, v\right)$ and $\left(y_{0}, b_{1}, w_{1}\right) \sim_{E}\left(t_{0}, p_{1}, v\right)$, by Lemma 3.3(i),

$$
\left(x_{0}^{2} y_{0}^{2}, b_{1}, w_{1}\right) \quad \sim_{E} \quad\left(q_{0}^{2} t_{0}^{2}, p_{1}, v\right) .
$$

But as $\left(a_{0}^{2}, a_{1}, v_{1}\right) \sim_{E}\left(x_{0}^{2}, b_{1}, w_{1}\right)$ and $\left(c_{0}^{2}, a_{1}, v_{1}\right) \sim_{E}\left(y_{0}^{2}, b_{1}, w_{1}\right)$, one has $\left(p_{0}^{2}, p_{1}, v\right)$ $\sim_{E}\left(q_{0}^{2}, p_{1}, v\right)$ and $\left(s_{0}^{2}, p_{1}, v\right) \sim_{E}\left(t_{0}^{2}, p_{1}, v\right)$. Since $\left(p_{0}^{2}, p_{1}, v\right) \sim_{E}\left(q_{0}^{2}, p_{1}, v\right)$ and $\left(s_{0}, p_{1}, v\right) \sim_{E}\left(s_{0}, p_{1}, v\right)$, by Lemma 3.3(i), $\left(p_{0}^{2} s_{0}^{2}, p_{1}, v\right) \sim_{E}\left(q_{0}^{2} s_{0}^{2}, p_{1}, v\right)$. Also as $\left(s_{0}^{2}, p_{1}, v\right) \sim_{E}\left(t_{0}^{2}, p_{1}, v\right)$ and $\left(q_{0}, p_{1}, v\right) \sim_{E}\left(q_{0}, p_{1}, v\right)$, by Lemma 3.3(i), $\left(s_{0}^{2} q_{0}^{2}, p_{1}, v\right)$ $\sim_{E}\left(t_{0}^{2} q_{0}^{2}, p_{1}, v\right)$. Hence,

$$
\left(p_{0}^{2} s_{0}^{2}, p_{1}, v\right) \quad \sim_{E} \quad\left(t_{0}^{2} q_{0}^{2}, p_{1}, v\right)=\left(q_{0}^{2} t_{0}^{2}, p_{1}, v\right) .
$$

Thus by equations (1), (2) and (3),

$$
\left(a_{0}^{2} c_{0}^{2}, a_{1}, v_{1}\right) \sim_{E}\left(x_{0}^{2} y_{0}^{2}, b_{1}, w_{1}\right) .
$$

Now suppose $r$ is odd. By Mennicke-Newman Lemma 3.1, one can arrange

$$
\begin{array}{ll}
\left(a_{0}, a_{1}, v_{1}\right) \sim_{E}\left(p_{0}, p_{1}, v\right), & \left(x_{0}, b_{1}, w_{1}\right) \sim_{E}\left(q_{0}, p_{1}, v\right), \\
\left(c_{0}, a_{1}, v_{1}\right) \sim_{E}\left(s_{0}, p_{1}, v\right) \text { and } & \left(y_{0}, b_{1}, w_{1}\right) \sim_{E}\left(t_{0}, p_{1}, v\right) .
\end{array}
$$

By Lemma 2.2, one has

$$
\begin{array}{ll}
\left(a_{0}, a_{1}^{2}, v_{1}\right) \sim_{E}\left(p_{0}, p_{1}^{2}, v\right), & \left(x_{0}, b_{1}^{2}, w_{1}\right) \sim_{E}\left(q_{0}, p_{1}^{2}, v\right) \\
\left(c_{0}, a_{1}^{2}, v_{1}\right) \sim_{E}\left(s_{0}, p_{1}^{2}, v\right) \text { and } \quad\left(y_{0}, b_{1}^{2}, w_{1}\right) \sim_{E}\left(t_{0}, p_{1}^{2}, v\right)
\end{array}
$$

and

$$
\begin{array}{ll}
\left(a_{0}^{2}, a_{1}^{2}, v_{1}\right) \sim_{E}\left(p_{0}^{2}, p_{1}^{2}, v\right), & \left(x_{0}^{2}, b_{1}^{2}, w_{1}\right) \sim_{E}\left(q_{0}^{2}, p_{1}^{2}, v\right) \\
\left(c_{0}^{2}, a_{1}^{2}, v_{1}\right) \sim_{E}\left(s_{0}^{2}, p_{1}^{2}, v\right) \text { and } & \left(y_{0}^{2}, b_{1}^{2}, w_{1}\right) \sim_{E}\left(t_{0}^{2}, p_{1}^{2}, v\right)
\end{array}
$$


Since $\left(a_{0}^{2}, a_{1}^{2}, v_{1}\right) \sim_{E}\left(p_{0}^{2}, p_{1}^{2}, v\right)$ and $\left(c_{0}, a_{1}^{2}, v_{1}\right) \sim_{E}\left(s_{0}, p_{1}^{2}, v\right)$, one has by Lemma 3.3 (ii),

$$
\left(a_{0}^{2} c_{0}^{2}, a_{1}^{2}, v_{1}\right) \quad \sim_{E} \quad\left(p_{0}^{2} s_{0}^{2}, p_{1}^{2}, v\right) .
$$

Also as $\left(x_{0}^{2}, b_{1}^{2}, w_{1}\right) \sim_{E}\left(q_{0}^{2}, p_{1}^{2}, v\right)$ and $\left(y_{0}, b_{1}^{2}, w_{1}\right) \sim_{E}\left(t_{0}, p_{1}^{2}, v\right)$, by Lemma 3.3(ii),

$$
\left(x_{0}^{2} y_{0}^{2}, b_{1}^{2}, w_{1}\right) \quad \sim_{E} \quad\left(q_{0}^{2} t_{0}^{2}, p_{1}^{2}, v\right) .
$$

But as $\left(a_{0}^{2}, a_{1}^{2}, v_{1}\right) \sim_{E}\left(x_{0}^{2}, b_{1}^{2}, w_{1}\right)$ and $\left(c_{0}^{2}, a_{1}^{2}, v_{1}\right) \sim_{E}\left(y_{0}^{2}, b_{1}^{2}, w_{1}\right)$, one has $\left(p_{0}^{2}, p_{1}^{2}, v\right)$ $\sim_{E}\left(q_{0}^{2}, p_{1}^{2}, v\right)$ and $\left(s_{0}^{2}, p_{1}^{2}, v\right) \sim_{E}\left(t_{0}^{2}, p_{1}^{2}, v\right)$. Since $\left(p_{0}^{2}, p_{1}^{2}, v\right) \sim_{E}\left(q_{0}^{2}, p_{1}^{2}, v\right)$ and $\left(s_{0}, p_{1}^{2}, v\right) \sim_{E}\left(s_{0}, p_{1}^{2}, v\right)$, by Lemma 3.3(ii), $\left(p_{0}^{2} s_{0}^{2}, p_{1}^{2}, v\right) \sim_{E}\left(q_{0}^{2} s_{0}^{2}, p_{1}^{2}, v\right)$. Also as $\left(s_{0}^{2}, p_{1}^{2}, v\right) \sim_{E}\left(t_{0}^{2}, p_{1}^{2}, v\right)$ and $\left(q_{0}, p_{1}^{2}, v\right) \sim_{E}\left(q_{0}, p_{1}^{2}, v\right)$, by Lemma 3.3(ii), $\left(s_{0}^{2} q_{0}^{2}, p_{1}^{2}, v\right)$ $\sim_{E}\left(t_{0}^{2} q_{0}^{2}, p_{1}^{2}, v\right)$. Hence,

$$
\left(p_{0}^{2} s_{0}^{2}, p_{1}^{2}, v\right) \quad \sim_{E} \quad\left(t_{0}^{2} q_{0}^{2}, p_{1}^{2}, v\right)=\left(q_{0}^{2} t_{0}^{2}, p_{1}^{2}, v\right) .
$$

Thus by equations (4), (5) and (6),

$$
\left(a_{0}^{2} c_{0}^{2}, a_{1}^{2}, v_{1}\right) \sim_{E}\left(x_{0}^{2} y_{0}^{2}, b_{1}^{2}, w_{1}\right)
$$

as required.

Following the terminology in [5], we say the group structure on the orbit space $\mathrm{Um}_{r+1}(A) / \mathrm{E}_{r+1}(A), r \geq 2$, is nice if

$$
\left[\left(a, z_{1}, \ldots, z_{r}\right)\right] *\left[\left(b, z_{1}, \ldots, z_{r}\right)\right]=\left[\left(a b, z_{1}, \ldots, z_{r}\right)\right],
$$

for all $\left(a, z_{1}, \ldots, z_{r}\right),\left(b, z_{1}, \ldots, z_{r}\right) \in \mathrm{Um}_{r+1}(A)$.

Proposition 3.5. Let $A$ be an affine algebra of dimension d over an algebraically closed field $k$, with characteristic $k \neq 2$. Then the group structure on the orbit space $\mathrm{Um}_{r+1}(A) / \mathrm{E}_{r+1}(A)$ is nice if $r=d$. If $k$ is the algebraic closure of a finite field, then the group structure on the orbit space is also nice when $r=d-1 \geq 2$.

Proof. By Swan's version of the Bertini Theorem (see [16]),

$$
\begin{aligned}
{\left[\left(a, z_{1}, \ldots, z_{d}\right)\right] } & =\left[\left(a, z_{1}^{\prime}, \ldots, z_{d}^{\prime}\right)\right], \\
{\left[\left(b, z_{1}, \ldots, z_{d}\right)\right] } & =\left[\left(b, z_{1}^{\prime}, \ldots, z_{d}^{\prime}\right)\right]
\end{aligned}
$$

for some $z_{1}^{\prime}, \ldots, z_{d}^{\prime} \in A$, with $z_{i}^{\prime}-z_{i} \in A a b$, for $1 \leq i \leq d$, and with $\operatorname{ht}\left(z_{1}^{\prime}, \ldots, z_{d}^{\prime}\right)=$ $d$. Now $\left(A /\left(z_{1}^{\prime}, \ldots, z_{d}^{\prime}\right)\right)_{\text {red }}=k \times \cdots \times k$. Since $k$ is algebraically closed, and $1 / 2 \in R$, we can write $a=c^{4}$ modulo $\left(z_{1}^{\prime}, \ldots, z_{d}^{\prime}\right)$, for some $c \in R$, and $b=$ $d^{4}$ modulo $\left(z_{1}^{\prime}, \ldots, z_{d}^{\prime}\right)$, for some $d \in R$. Consequently,

$$
\begin{aligned}
{\left[\left(a b, z_{1}, \ldots, z_{d}\right)\right] } & =\left[\left(a b, z_{1}^{\prime}, \ldots, z_{d}^{\prime}\right)\right] \\
& =\left[\left(c^{4} d^{4}, z_{1}^{\prime}, \ldots, z_{d}^{\prime}\right)\right] \\
& =\left[\left(c^{4}, z_{1}^{\prime}, \ldots, z_{d}^{\prime}\right)\right] *\left[\left(d^{4}, z_{1}^{\prime}, \ldots, z_{d}^{\prime}\right)\right] \\
& =\left[\left(a, z_{1}^{\prime}, \ldots, z_{d}^{\prime}\right)\right] *\left[\left(b, z_{1}^{\prime}, \ldots, z_{d}^{\prime}\right)\right] \\
& =\left[\left(a, z_{1}, \ldots, z_{d}\right)\right] *\left[\left(b, z_{1}, \ldots, z_{d}\right)\right] .
\end{aligned}
$$

Done. The proof is similar to the above proof in the case when $k$ is the algebraic closure of a finite field $k$. The only change is that we appeal to ([15], Theorem 17.2) instead of Swan's version of the Bertini Theorem. The rest of the argument is identical.

\section{ACKNOWLEDGMENTS}

We thank the referee for overlooking our "peccadillos", and also for helping us to get a correct proof of Lemma 3.4. 


\section{REFERENCES}

[1] J.F. AdAms, Vector fields on spheres. Ann. of Math. (2), 75, 603-632 (1962). MR0139178 $(25: 2614)$

[2] H. BAss, Algebraic K-theory. W. A. Benjamin, Inc., New York-Amsterdam (1968). MR0249491 (40:2736)

[3] S.M. Bhatwadekar and Raja Sridharan, The Euler class group of a Noetherian ring. Compositio Math. 122, no. 2, 183-222 (2000). MR1775418 (2001g:13018)

[4] S.M. Bhatwadekar and Raja Sridharan, On Euler classes and stably free projective modules. Algebra, arithmetic and geometry, Part I, II (Mumbai, 2000), 139-158, Tata Inst. Fund. Res. Stud. Math. 16, Tata Inst. Fund. Res., Bombay, 2002. MR1940666 (2003i:13012)

[5] A.S. GAdre and R.A. RAO, A nice group structure on the orbit space of unimodular rows, (preprint 2006), accepted and to appear in K-theory, January 2008.

[6] S. Jose and R.A. RaO, A structure theorem for the Elementary Unimodular Vector group, Trans. Amer. Math. Soc. 358, no.7, 3097-3112 (2005). MR2216260 (2007a:20047)

[7] S. Jose and R.A. RAO, A local global principle for the elementary unimodular vector group, Commutative Algebra and Algebraic Geometry (Bangalore, India, 2003), 119-125, Contemp. Math. 390, Amer. Math. Soc., Providence, RI (2005). MR2187329 (2006h:19001)

[8] S. Jose and R.A. RAO, A Fundamental Property of Suslin matrices, preprint 2007.

[9] R.A. RAO, The Bass-Quillen conjecture in dimension three but characteristic $\neq 2,3$ via a question of A. Suslin. Invent. Math. 93, 3, 609-618 (1988). MR952284 (89d:13011)

[10] R.A. RaO, On completing unimodular polynomial vectors of length three. Trans. Amer. Math. Soc. 325, no. 1, 231-239 (1991). MR991967 (91h:13008)

[11] R.A. RAO and W. VAN DER KALLEN, Improved stability for $K_{1}$ and $\mathrm{WMS}_{d}$ of a nonsingular affine algebra. $K$-theory (Strasbourg, 1992). Asterisque no. 226, 11, 411-420 (1994). MR1317126 (96e:19001)

[12] R.A. RAO, An Abelian Group Structure on Orbits of "Unimodular Squares" in Dimension 3, Journal of Algebra 210, 216-224 (1998). MR1656421 (99k:20103)

[13] M. Roitman, On stably extended projective modules over polynomial rings. Proc. Amer. Math. Soc. 97, no. 4, 585-589 (1986). MR845969 (87f:13007)

[14] A.A. Sustin, On Stably Free Modules, Math. USSR Sbornik 31, 479-491 (1977). MR0441949 $(56: 340)$

[15] A.A. Suslin and L.N. VASERstein, Serre's problem on projective modules over polynomial rings and algebraic $K$-theory, Math. USSR Izv. 10, 937-1000 (1976). MR0447245 (56:5560)

[16] R.G. Swan, A cancellation theorem for projective modules in the metastable range. Invent. Math. 27, 23-43 (1974). MR0376681 (51:12856)

[17] L.N. VASERSTEIN, Operations on orbits of unimodular vectors, Journal of Algebra 100, 456461 (1986). MR840588 (87j:13018)

[18] L.N. Vaserstein, Stable rank and dimensionality of topological spaces, Funktsional. Anal. i Prilozhen. 5, 17-27 (1971), translation in Functional Anal. Appl. 5, 102-110, 1971. MR0284476 (44:1701)

[19] W. VAN DER KALLEN, A group structure on certain orbit sets of unimodular rows, Journal of Algebra 82, no. 2, 363-397 (1983). MR704762 (85b:13014)

[20] W. van DeR Kallen, A module structure on certain orbit sets of unimodular rows, J. Pure Appl. Algebra 57, 657-663 (1975). MR987316 (90b:18013)

[21] W. van Der Kallen, From Mennicke symbols to Euler class groups. Algebra, arithmetic and geometry, Part I, II (Mumbai, 2000), 341-354, Tata Inst. Fund. Res. Stud. Math. 16, Tata Inst. Fund. Res., Bombay (2002). MR1940672 (2003h:19006)

School of Mathematics, Tata Institute of Fundamental Research, Dr. Homi Bhabha Road, Mumbai, India 400005

E-mail address: ravi@math.tifr.res.in

Department of Mathematics, Ismail Yusuf College, Jogeshwari(E), Mumbai, India 400-060

E-mail address: selbyjose@rediffmail.com 